\title{
The effects of the printing direction and UV artificial degradation on the mechanical properties using AM PolyJet technology
}

\author{
M. Matušů ${ }^{a, *}$, D. Blaha ${ }^{a}$, P. David $^{a}$, Z. Padovec ${ }^{a}$, P. Růžička $^{a}$, J. Rezníček $^{a}$, \\ M. Růžička ${ }^{a}$ \\ ${ }^{a}$ Faculty of Mechanical Engineering, Czech Technical University in Prague, Technická 4, 16000 Praha, Czech Republic
}

Received 19 October 2020; accepted 7 May 2021

\begin{abstract}
This study addresses differences in the material properties due to the influence of the printing direction, UV degradation and the use of differently coloured materials. The specimens were manufactured employing PolyJet technology from the Vero material (VeroBlue, VeroYellow, VeroMagenta). Subsequently, the specimens were subjected to tension and compression testing. Ageing is simulated via the exposure of the specimens to solar UVA \& UVB for 15 and 30 days (the calculated daylight UV exposure for half a year and a whole year, respectively). The differences in the material properties were also determined according to the types of material. The results were then subjected to a statistical analysis.
\end{abstract}

(c) 2021 University of West Bohemia. All rights reserved.

Keywords: additive manufacturing, PolyJet, stereolithography apparatus, UV degradation, statistical analysis

\section{Introduction}

The demand for the rapid prototyping of products is increasing exponentially, in connection with which the number of procedures via which items can be produced using $3 \mathrm{D}$ printing is substantial. Additive manufacturing is capable of creating very complicated shapes with various cavities and difficult-to-reproduce curves [8]. The technologies that enable the printing of plastic materials include fused deposition modelling (FDM) [7,8], PolyJet photopolymer printing or the stereolithography apparatus (SLA) [6,8,9], laminated object manufacturing (LOM) [8] and selective laser sintering (SLS) [8].

PolyJet technology employs acrylic-based photopolymers, and the printing process consists of the jetting of layers of material which are then cured with UV light radiation so that the material is stacked layer by layer in the direction of the $z$-axis. Since the technology is able to print in layers of up to 16 microns, it is possible to print very precise shapes $[3,10]$. The quality of the product depends on several parameters, i.e., the exposure time and radiation intensity, which differ according to the material (Vero, Tango, etc.), the width of the support material between the stack-up products and the distance between the various printed components [6]. Moreover, the placement and rotation of objects printed using PolyJet technology affects the mechanical properties of the printed products [1]. UV curing occurs in the range $350 \mathrm{~nm}$ to $410 \mathrm{~nm}$, within which the radiation exerts the optimal impact on the photopolymer [2].

According to the materials list provided by Stratasys [10], there are no differences in terms of the tensile strength and Young's modulus of elasticity between products printed in differing

*Corresponding author. Tel.: +420 224352 519, e-mail: Martin.Matusu@fs.cvut.cz. https://doi.org/10.24132/acm.2021.649 
directions (according to ISO/ASTM 52921:2013 ZYX, YZX and YXZ directions). This factor forms the main subject of interest in this study.

The degradation of polymers during artificial ageing is simulated via exposure to UV radiation, which leads to changes in the molecular chains such as cross-linking and reductions in the molecular weight, which can be observed via changes in terms of mechanical testing and colour changes. The degree of the deterioration of the mechanical properties is significant when photopolymer-based products are exposed to UV spectra [11]. Since the photooxidation process affects particularly the surfaces of polymers, the thickness of the material comprises one of the most important factors in this respect.

Stratasys produces a wide range of materials for PolyJet technology, for example Tango (a rubber-like material), VeroFlex (a flexible material), VeroUltraClear and many other materials that are suited to PolyJet technology [10].

Stratasys does not provide any data on products printed in differing directions, and no information is available as to whether the application of sunlight is harmful to acrylic-based photopolymer products printed on PolyJet printers with respect to the mechanical properties.

\section{Preparation for testing}

Our main objective was to ascertain the mechanical properties of the printed components in various printing directions. The tension specimens were fabricated according to the ČSN EN ISO 527-1 standard [5] and a non-normalized shape for compression testing. The above standard sets out the tensile testing of plastic specimens and the evaluation of their mechanical properties, and is identical to the EN ISO 527-1 standard. Artificial ageing was induced under laboratory conditions using a Solar UVA \& UVB $80 \mathrm{~W}$ lamp for 15 days and 30 days according to the ČSN EN ISO 4892-3 standard [4], which sets out the procedure for the testing of specimens with fluorescent UV radiation, the respective measurement conditions and the use of UV lamps.

\subsection{Print modes}

All the tension specimens were printed in three different directions (ZYX, YZX and YXZ according to ISO/ASTM 52921:2013 — see Figs. 1 and 4) for each material (VeroBlue, VeroYellow and VeroMagenta - see Fig. 2) with areas of $40 \mathrm{~mm}^{2}$, widths of $10.00 \pm 0.11 \mathrm{~mm}$ and thicknesses of $3.99 \pm 0.09 \mathrm{~mm}$. One group of specimens, designated L, was printed as YXZ, the second group of specimens, designated B, was printed as YZX and the third group of specimens, designated $\mathrm{S}$, was printed as ZYX.

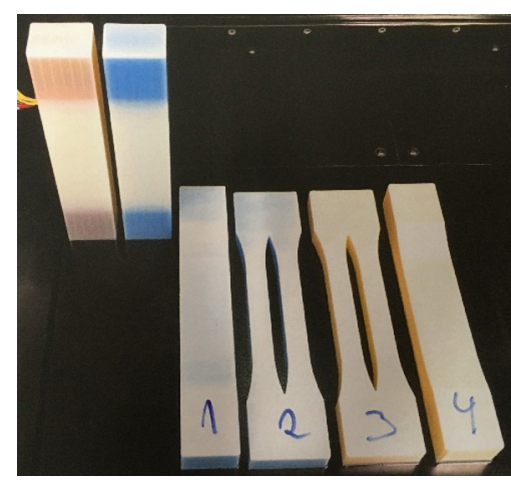

Fig. 1. From left: two S, B $(1,4)$ and $L(2,3)$ specimens; all the specimens are covered in a support material

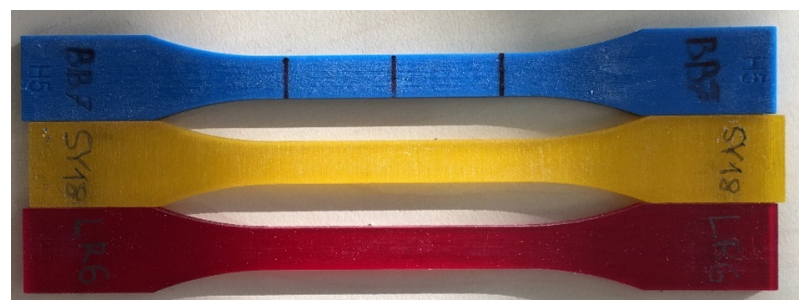

Fig. 2. Demonstration of the various colours and structures (VeroBlue, VeroYellow, VeroMagenta) 
The compression specimens (Fig. 3) were printed in the form of small cylinders with diameters of $12 \mathrm{~mm}$ and heights of $24 \mathrm{~mm}$. These specimens were printed as ZXY, designated LV, and YZX, designated SV, for each of the materials (VeroBlue, VeroYellow, VeroMagenta).

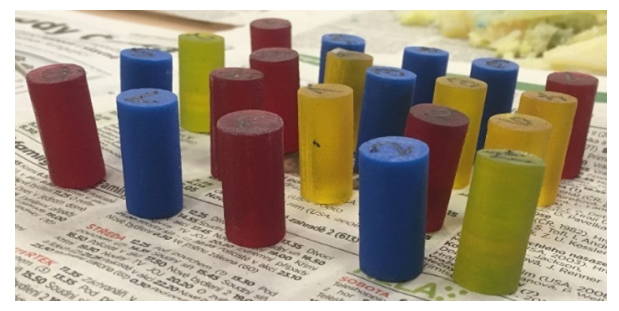

Fig. 3. Compression specimens printed vertically (ZXY); all colours

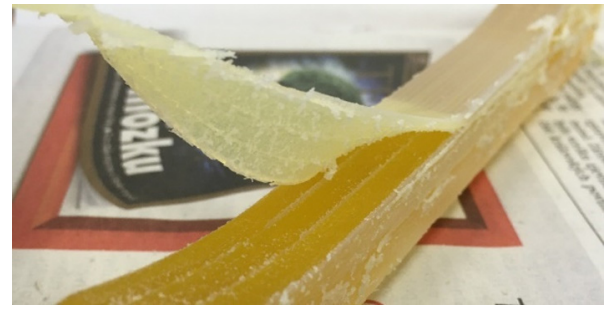

Fig. 4. Removal of the support material from three tension specimens

\subsection{Testing setup}

All the test specimens were subjected to loading on a TIRA Test 2300 universal testing machine with a $20 \mathrm{kN}$ load cell. The tension specimens were measured using a UTS Testsystem GmbH external strain gauge (Fig. 5). The compression specimens were tested without an external strain gauge, see Fig. 6.

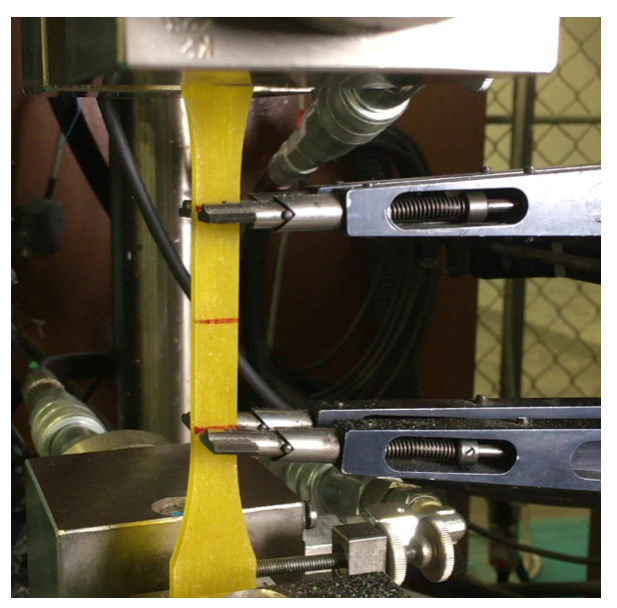

Fig. 5. Attachment of the external strain gauge

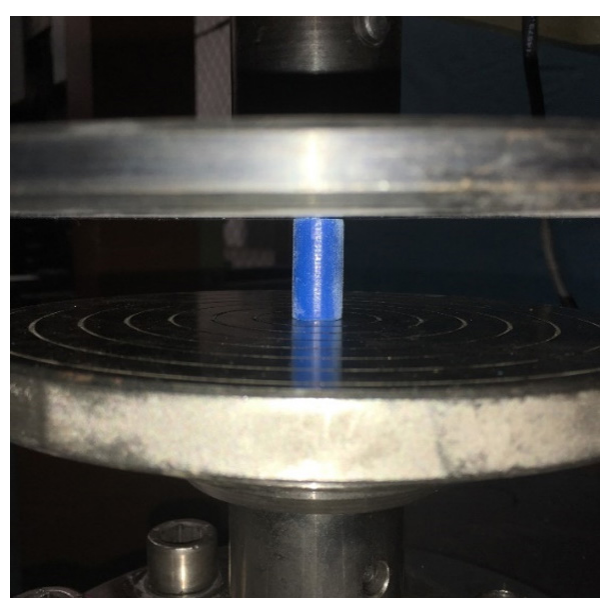

Fig. 6. Compression specimen

\subsection{UV set-up}

A solar UVA \& UVB $80 \mathrm{~W}$ lamp was used in the chamber for the UV degradation (Fig. 8). The specimens were placed at a distance of $50 \mathrm{~cm}$ from the UV lamp with a fluence rate of $60 \mu \mathrm{W} / \mathrm{cm}^{2}$ for the UVA radiation and $67 \mu \mathrm{W} / \mathrm{cm}^{2}$ for the UVB radiation. The temperature of the samples was kept constant throughout the experiment at $25 \pm 1^{\circ} \mathrm{C}$ (Fig. 7).

\section{Testing of the specimens}

\subsection{Tensile specimens}

All the tensile specimens were tested following the specimen preparation phase. The test results indicated that the Young's modulus of elasticity differed slightly according to the printing 


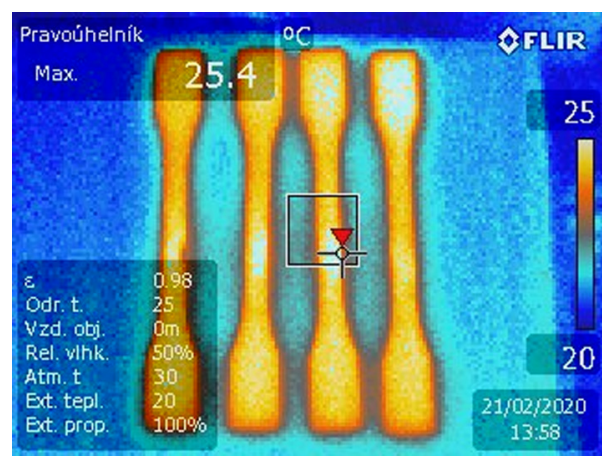

Fig. 7. Image taken by the Flir A315 thermal camera

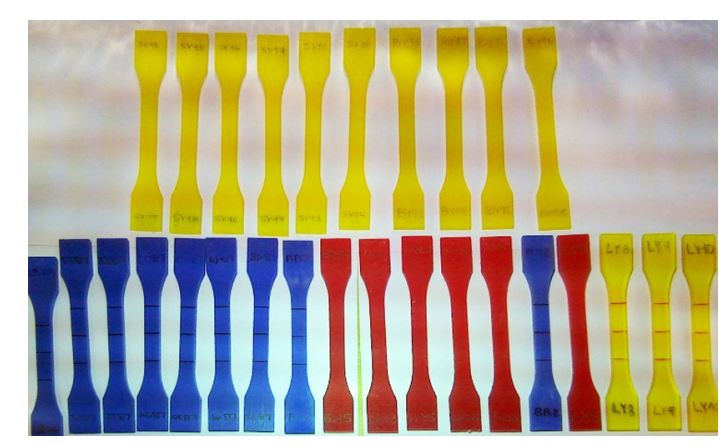

Fig. 8. Specimens exposed to radiation in the laboratory

direction and the material. The modulus differed for each printing direction and material. The main differences between the Young's moduli of elasticity were in a range of around 5\%, as shown in Figs. 9-11. The statistical analysis considered all the printing modes and materials.

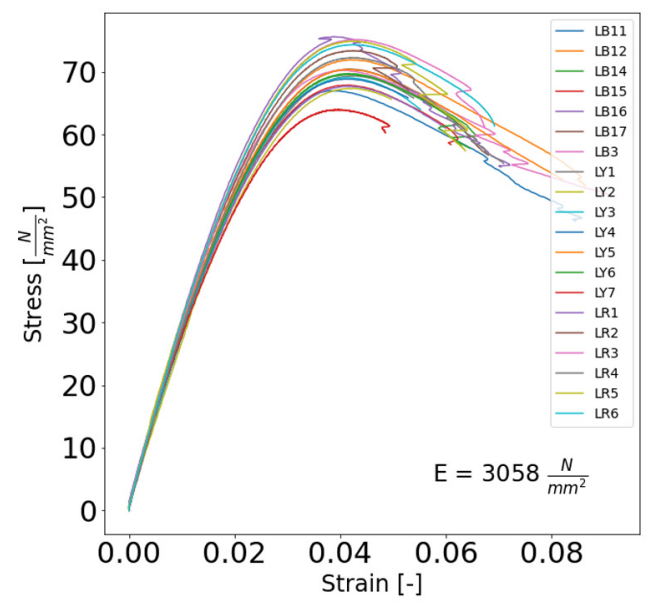

Fig. 9. Specimens of the materials printed in the horizontal direction — L-specimens (YXZ)

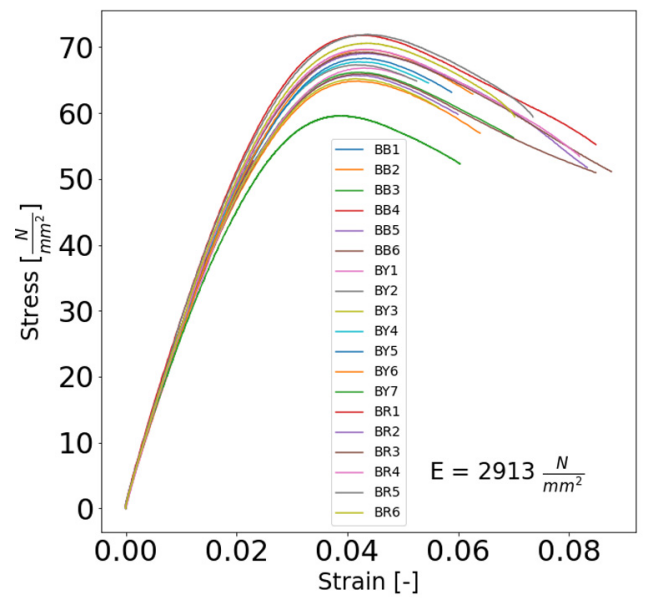

Fig. 10. Specimens of the materials printed in the horizontal direction — B-specimens (YZX)

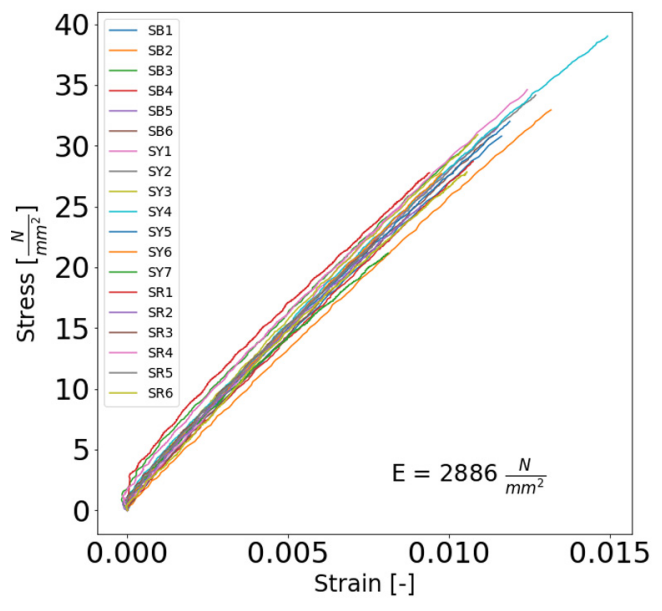

Fig. 11. Specimens of the materials printed in the vertical direction $-\mathrm{S}$-specimens $(\mathrm{ZYX})$ 


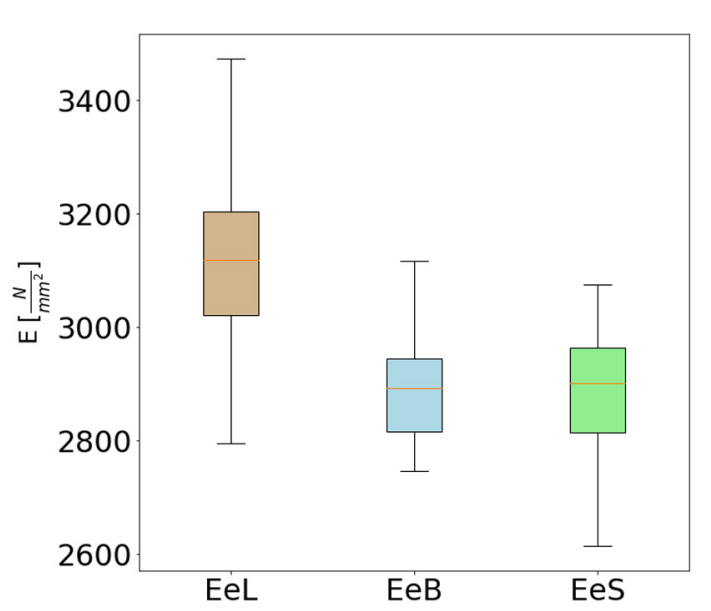

Fig. 12. Comparison of the E modulus with respect to the direction of printing; from left to right $\mathrm{L}, \mathrm{B}, \mathrm{S}$

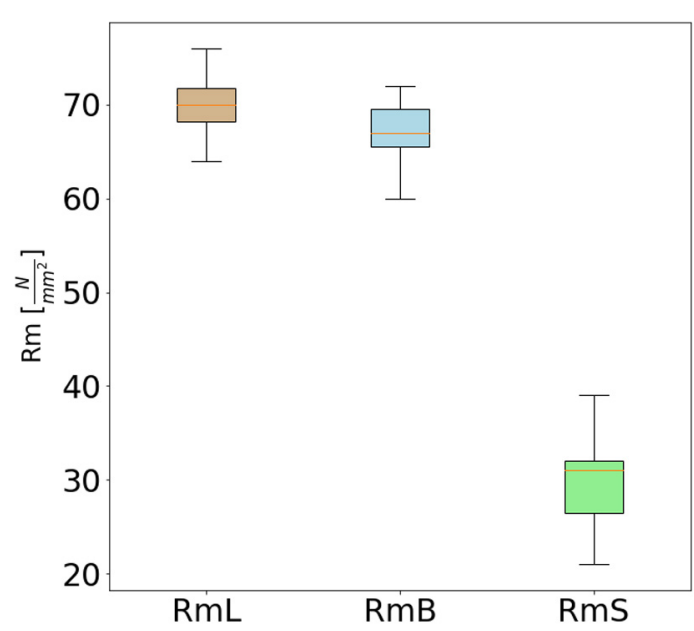

Fig. 13. Comparison of the ultimate strength stress in tension with respect to the direction of printing; from left to right $\mathrm{L}, \mathrm{B}, \mathrm{S}$

The differences in the moduli of elasticity are significantly more evident from the box plot (Fig. 12), which indicates the differences in the median of the modulus; the highest modulus of elasticity was observed for the L-specimens.

In contrast, the ultimate strength stress in tension differed visibly between the horizontally printed (L, B) specimens and the vertically printed specimens $(\mathrm{S})$. A small plasticity area prior to fracture was visible with respect to the L- and B-specimens, whereas the S-specimens behaved as brittle materials and were unable to withstand the same force exerted in the tensile tests as did the L- and B-specimens (Fig. 13).

A further experimental objective concerned the determination of whether materials from the same family (Vero) behaved differently according to the differing pigmentation levels. While, according to the Stratasys material data sheet [10], VeroYellow and VeroMagenta are identical, the VeroBlue material evinces different properties.

Although small differences were observed between the moduli of elasticity of all the materials, from the statistical point of view, it is evident that the VeroMagenta specimens evince the highest moduli of elasticity and the VeroBlue materials the lowest moduli of elasticity (Fig. 14).

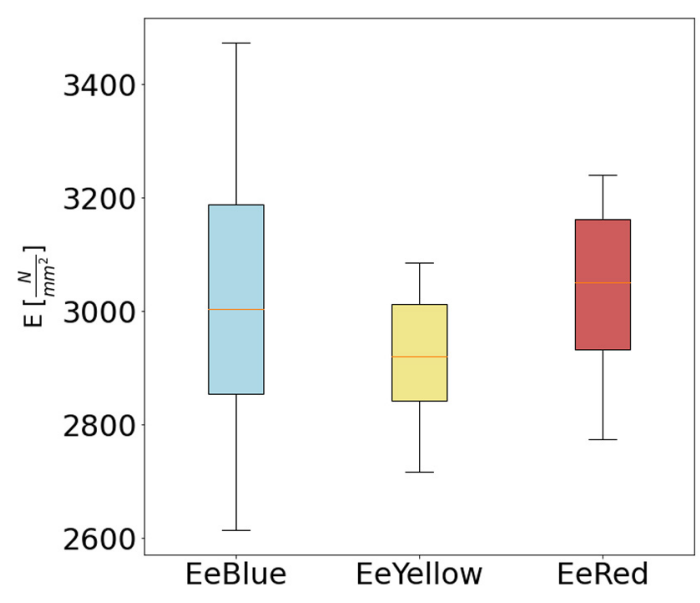

Fig. 14. Comparison of the E moduli of the printed materials; from left to right VeroBlue, VeroYellow, VeroMagenta 
Since the ultimate tensile strength is closely related to the direction of printing, the differences between the Vero materials must be considered separately rather than as a group that includes all the printing directions. The orientation of one of the printing directions illustrates the differences between the ultimate strength stress in tension of the materials (Fig. 15), thus demonstrating that the various materials behave differently.

The lowest ultimate strength was recorded for the VeroMagenta specimens printed in the vertical direction (RmS VeroMagenta) (Fig. 16), which was (according to the mean value) more than $25 \%$ lower than for the VeroYellow and VeroBlue materials. In contrast, the VeroMagenta material behaved differently in the B and L directions.

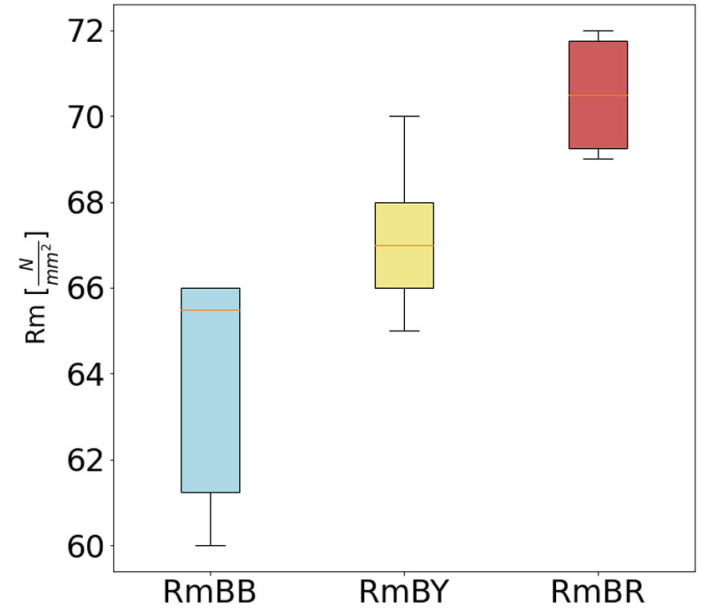

Fig. 15. Comparison of the ultimate strength stress in tension of the $\mathrm{B}$ printed material; from left to right VeroBlue, VeroYellow, VeroMagenta

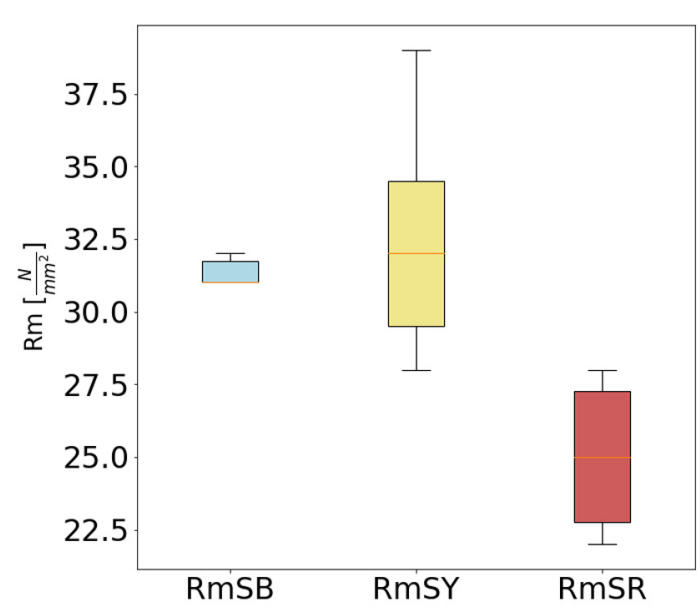

Fig. 16. Specimens printed in the vertical direction - S-specimens

\subsection{Statistical testing of distributions}

Since the box plot and mean and median results failed to clearly highlight the various differences, the statistical testing of the distributions was selected as an unbiased approach to the evaluation of the results of the tensile testing of the various materials and printing directions.

Firstly, all the distributions (E, Rm) were examined in order to determine whether they could be considered as normal distributions (Gaussian). The Shapiro-Wilk's test was used with a value of $\alpha=0.05$ for the examination of the type of distribution.

The $t$-test was used for two independent specimens in the case of the comparison of two specimens with Gaussian distributions, and the Mann-Whitney $U$-test was applied if at least one of the distributions was not normal. For example, the $R m_{L}$ (the ultimate strength stress for the tension specimen with an L printing orientation) was normally distributed while the $R m_{B}$ (ultimate strength stress for the tension specimen with a B printing orientation) was non-normally distributed, hence the $U$-test was applied.

Tables 1 and 2 indicate whether or not the distributions differed significantly: "YES" signifies significantly different, "NO" indicates non-significantly different. The significant differences shown in Tables 1 and 2 were confirmed for the distribution of the ultimate strength in tension for all the samples regardless of the printing direction. Even though the differences between $E_{L}$ (the Young's modulus for the samples printed in direction $\mathrm{L}$ ) and $E_{B}$ (the Young's modulus for the samples printed in direction B) were not visible from the box plot (Fig. 13), they 
Table 1. Comparison of the distributions with respect to the printing orientation

\begin{tabular}{|l|c|c|c|c|c|c|}
\hline & $E_{L}$ & $E_{B}$ & $E_{S}$ & $R m_{L}$ & $R m_{B}$ & $R m_{S}$ \\
\hline$E_{L}$ & & YES & YES & & & \\
\hline$E_{B}$ & YES & & NO & & & \\
\hline$E_{S}$ & YES & NO & & & & \\
\hline$R m_{L}$ & & & & & YES & YES \\
\hline$R m_{B}$ & & & & YES & & YES \\
\hline$R m_{S}$ & & & & YES & YES & \\
\hline
\end{tabular}

Table 2. Comparison of the distributions with respect to the printed material

\begin{tabular}{|c|c|c|c|}
\hline & $E_{B}$ & $E_{Y}$ & $E_{R}$ \\
\hline$E_{B}$ & & YES & YES \\
\hline$E_{Y}$ & YES & & YES \\
\hline$E_{R}$ & YES & YES & \\
\hline
\end{tabular}

were significantly different according to the statistical evaluation. However, the difference between $E_{B}$ and $E_{S}$ was not so significant. The differences between the types of materials were confirmed for all the distributions of the Young's modulus of elasticity. Moreover, the ultimate strength in tension was observed to differ significantly between the colours, even without the performance of the statistical testing, as shown in Figs. 15 and 16.

\subsection{The tensile specimens following artificial ageing}

The comparison of all the artificially aged and non-aged specimens revealed the higher degree of consistency of the spread of the values of the degraded specimens, which may have been due to the additional degree of drying during exposure to UV radiation under a constant temperature of $25 \pm 1^{\circ} \mathrm{C}$.

The degradation process did not result in visible changes in the Young's modulus of elasticity in any of the orientations, as can be seen from the stress-strain graphs in Figs. 17-19. However,

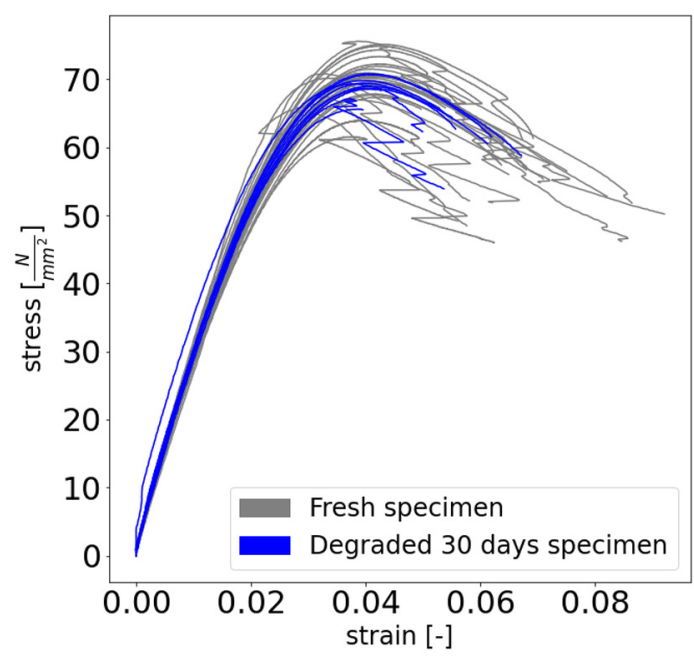

Fig. 17. Specimens printed in the horizontal direction — L-specimens 


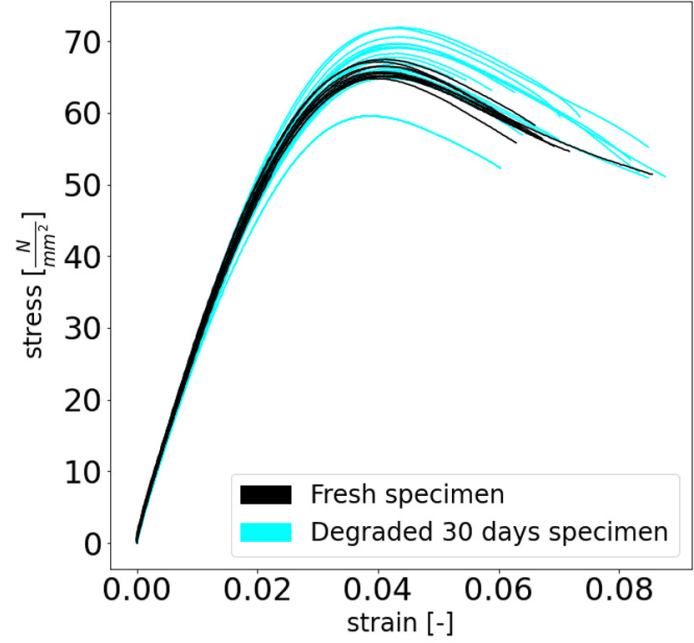

Fig. 18. Specimens printed in the horizontal direction - B-specimens

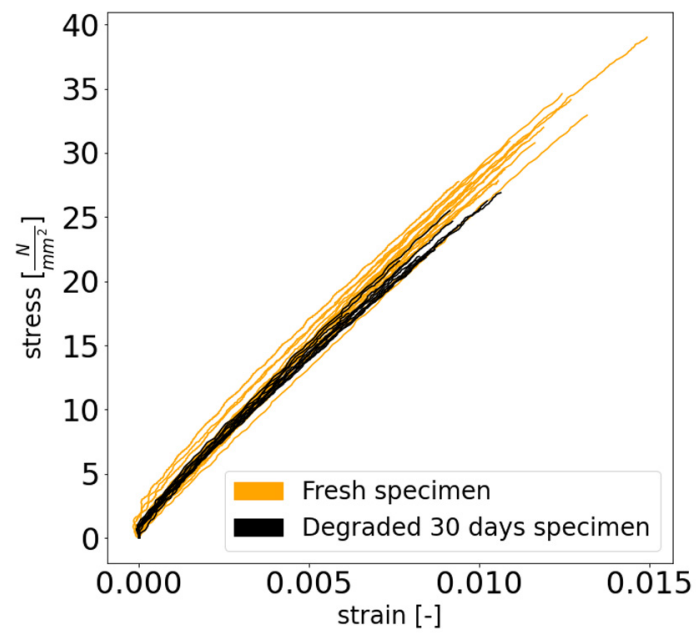

Fig. 19. Specimens printed in the vertical direction - S-specimens

the ultimate strength stress was lower in the B and L orientations and significantly lower in the S orientation.

The overall comparison of the ultimate strength stress and the Young's modulus of elasticity is significantly clearer from the boxplots (Figs. 20-24). The Young's modulus increased slightly with the degradation time with respect to the L orientation (Fig. 20), while it decreased slightly for the $\mathrm{S}$ orientation (Fig. 21). The magnitude of the elasticity modulus changes was insignificant compared to that of the ultimate strength stress during the artificial ageing process.

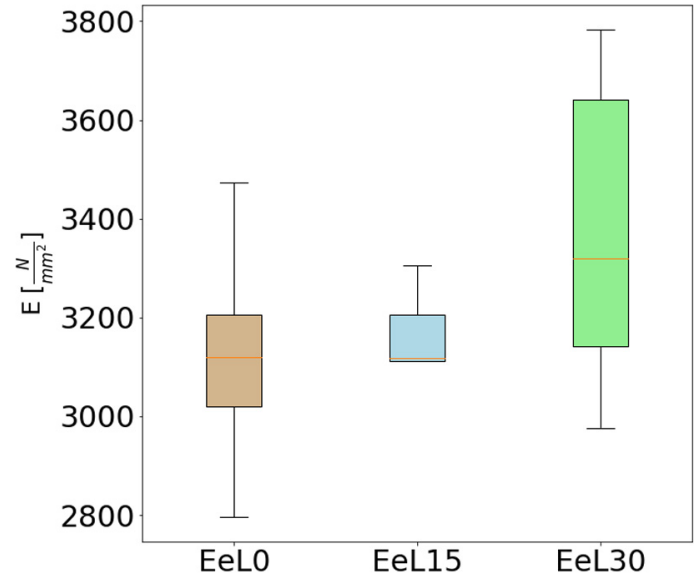

Fig. 20. Comparison of the E modulus of the non-degraded and degraded specimens after 15 and 30 days for the $\mathrm{L}$ orientation

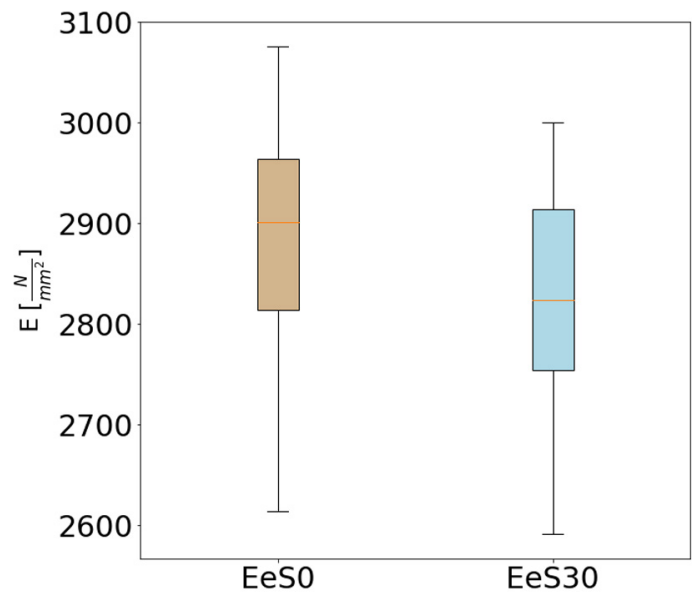

Fig. 21. Comparison of the E modulus of the non-degraded and degraded specimens after 30 days for the $\mathrm{S}$ orientation

The ultimate strength stress before and after artificial ageing was evaluated for each orientation: horizontal L (YXZ), see Fig. 23, side horizontal B (YZX), see Fig. 22, and vertical S (ZYX). All the orientations exhibited a descending tendency with respect to the ultimate strength stress. The differences between the $\mathrm{L}$ and $\mathrm{B}$ orientations during degradation were in single units of percent, in contrast to the $S$ orientation, where the artificial ageing process led to a change of nearly $30 \%$. Therefore, the accumulation of damage processes was significantly higher in the vertical orientation. 


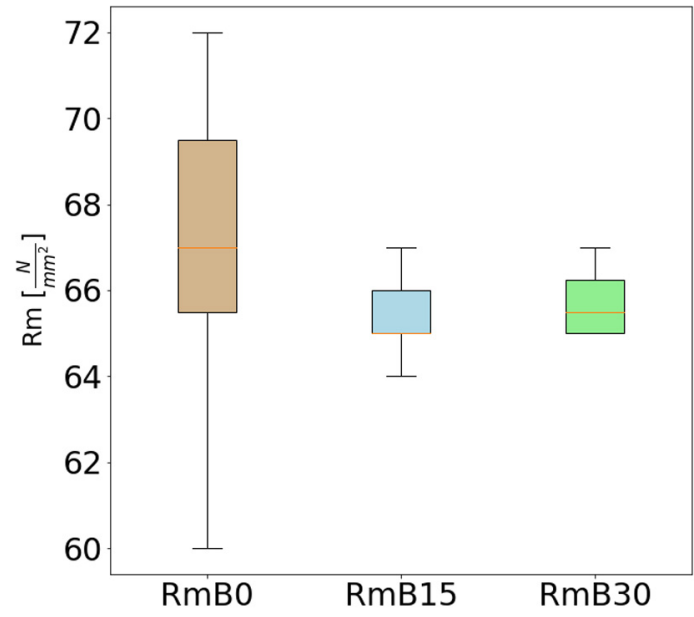

Fig. 22. Comparison of the ultimate strength stress of the non-degraded and degraded specimens after 15 and 30 days for the B orientation

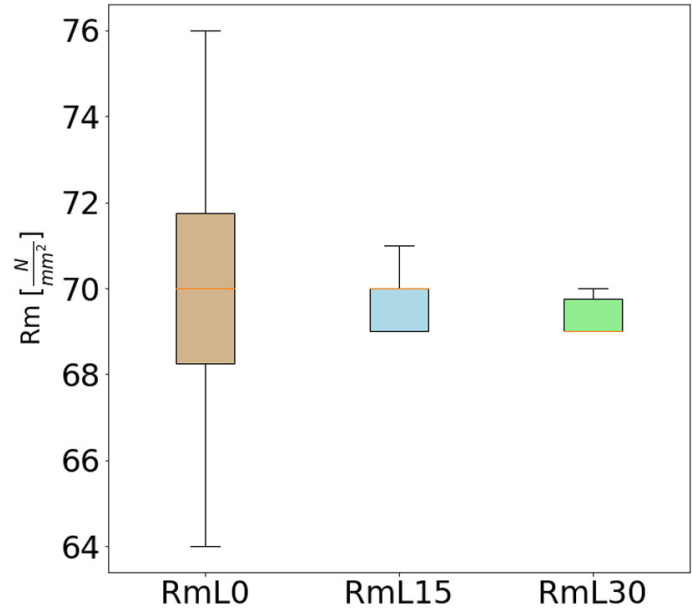

Fig. 23. Comparison of the ultimate strength stress of the non-degraded and degraded specimens after 15 and 30 days for the $\mathrm{L}$ orientation

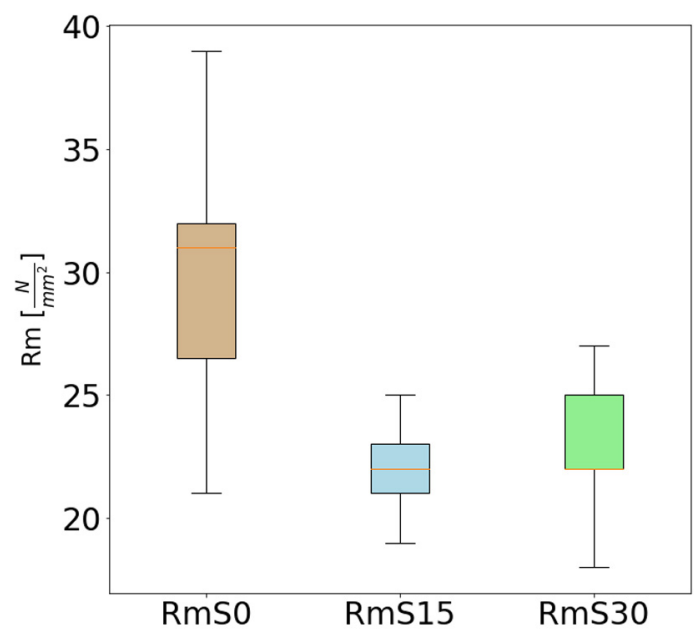

Fig. 24. Comparison of the ultimate strength stress of the non-degraded and degraded specimens after 15 and 30 days for the $\mathrm{S}$ orientation

\subsection{Statistical testing of the distributions following artificial ageing}

The degree of significance of the changes that occurred during the artificial ageing process as indicated by the Young's modulus and the ultimate strength stress was determined via the statistical evaluation approach for the tensile specimens only.

For example, Fig. 21, which considers the non-degraded and degraded S specimens after 30 days, illustrates slight differences with observable degradation, whereas, according to the statistical evaluation, the difference is not significant for the Young's modulus, as shown in Table 3. This is in contrast to the ultimate strength stress, concerning which the difference was significant during the artificial ageing process, but only after 30 days, as evident from Table 4.

The results clearly indicate significant differences between the samples. For the sake of clarity, Tables 3 and 4 indicate whether the differences between the two distributions were significant or not via a simple "YES" or "NO" entry. 
Table 3. Comparison of the distributions for the printing orientation

\begin{tabular}{|l|c|c|c|c|c|c|c|c|}
\hline$E$ & $L_{0}$ & $L_{15}$ & $L_{30}$ & $B_{0}$ & $B_{15}$ & $B_{30}$ & $S_{0}$ & $S_{30}$ \\
\hline$L_{0}$ & & NO & YES & & & & & \\
\hline$L_{15}$ & NO & & NO & & & & & \\
\hline$L_{30}$ & YES & NO & & & & & & \\
\hline$B_{0}$ & & & & & YES & YES & & \\
\hline$B_{15}$ & & & & YES & & YES & & \\
\hline$B_{30}$ & & & & YES & YES & & & \\
\hline$S_{0}$ & & & & & & & & NO \\
\hline$S_{30}$ & & & & & & & NO & \\
\hline
\end{tabular}

Table 4. Comparison of the distributions for the printed materials

\begin{tabular}{|l|l|l|l|l|l|l|l|l|l|}
\hline$R m$ & $L_{0}$ & $L_{15}$ & $L_{30}$ & $B_{0}$ & $B_{15}$ & $B_{30}$ & $S_{0}$ & $S_{15}$ & $S_{30}$ \\
\hline$L_{0}$ & & NO & NO & & & & & & \\
\hline$L_{15}$ & NO & & NO & & & & & & \\
\hline$L_{30}$ & NO & NO & & & & & & & \\
\hline$B_{0}$ & & & & & NO & YES & & & \\
\hline$B_{15}$ & & & & NO & & NO & & & \\
\hline$B_{30}$ & & & & YES & NO & & & & \\
\hline$S_{0}$ & & & & & & & & YES & YES \\
\hline$S_{15}$ & & & & & & & YES & & NO \\
\hline$S_{30}$ & & & & & & & YES & NO & \\
\hline
\end{tabular}

Table 4 shows that the significant differences relate to a greater extent to the samples that were degraded over 30 days (simulated for one year), particularly with respect to the $\mathrm{S}$ orientation of the printed material.

\subsection{Compression specimens}

This part of the experiment involved the testing of compression specimens with two different printing orientations. The compression testing served for the evaluation of the ultimate compressive strength, which was observed to differ slightly (but not significantly) according to the printing direction and the material.

As mentioned previously, the slight changes in the material and the printing method are not clear from the stress/strain graphs; moreover, the differences are not clear even from the box plots since the mean value of the ultimate compressive strength was around $104 \mathrm{~N} \mathrm{~mm}^{-2}$ for all the specimens. Hence, the $t$-test and the $U$-test were applied to each pair of distributions, the results of which, as expected, also indicated no significant differences between either the direction of printing or the material.

The main differences were determined from the distribution of the ultimate strength values (Figs. 25 and 26). While the ultimate strength of the various materials differed slightly, the mean value was practically the same (Figs. 27 and 28). 


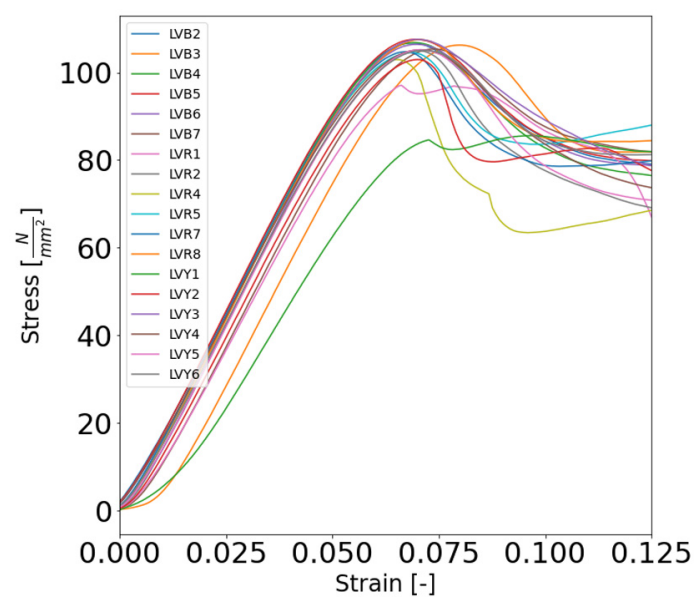

Fig. 25. Specimens printed in the horizontal direction; LV-specimens

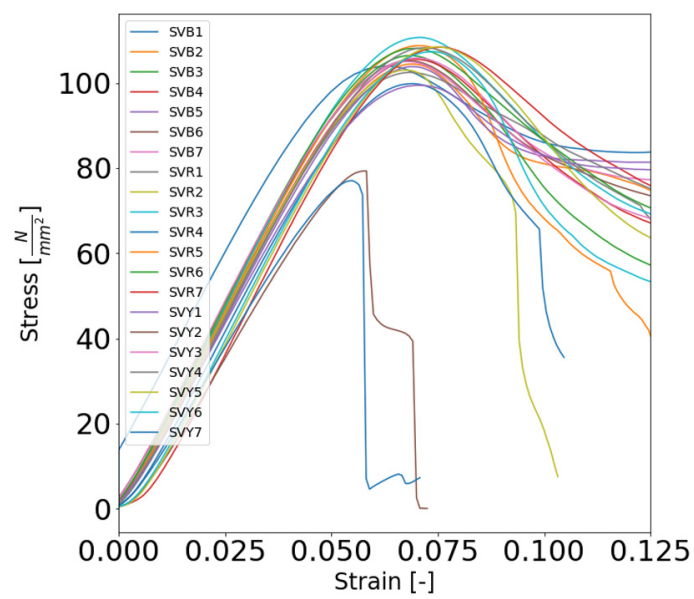

Fig. 26. Specimens printed in the vertical direction; SV-specimens

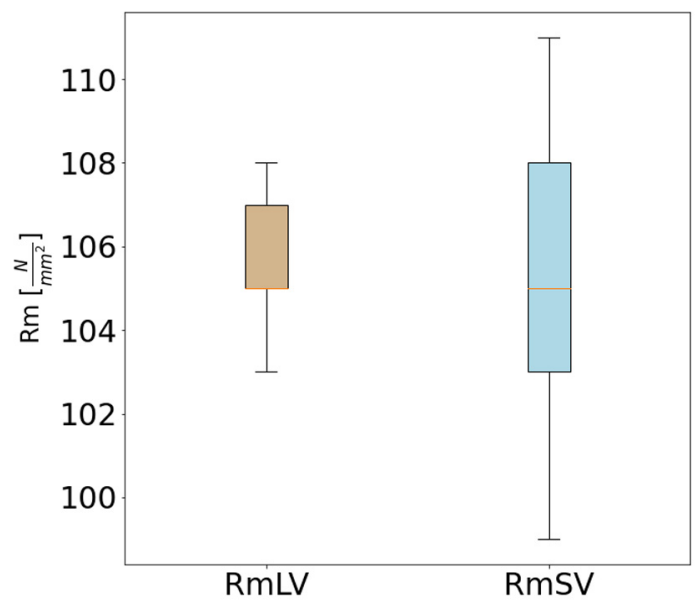

Fig. 27. Comparison of the ultimate strength from left to right (LV-specimens, SV-specimens)

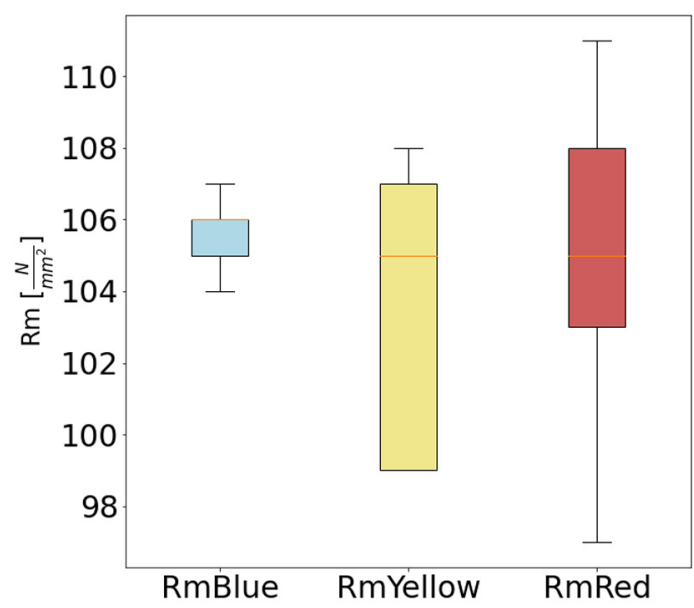

Fig. 28. Comparison of the ultimate strength from left to right (VeroBlue, VeroYellow, VeroMagenta) 


\subsection{Compression specimens following artificial ageing}

The degradation process caused by artificial ageing was also evaluated for the compression specimens. Unlike the tensile specimens, the compression samples were evaluated only for the various printing materials (not for the orientations). All the materials were printed in the LV horizontal orientation (ZYX).

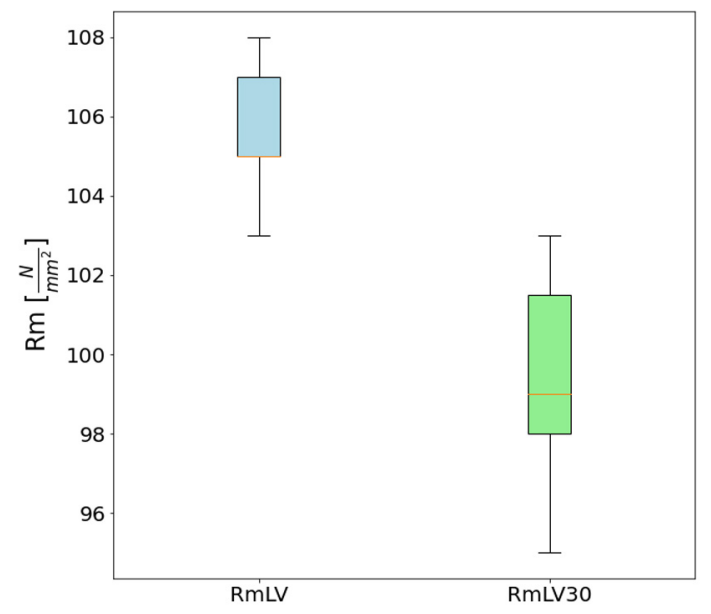

Fig. 29. Comparison of the ultimate strength stress of the non-degraded and degraded specimens after 30 days in the $\mathrm{L}$ orientation

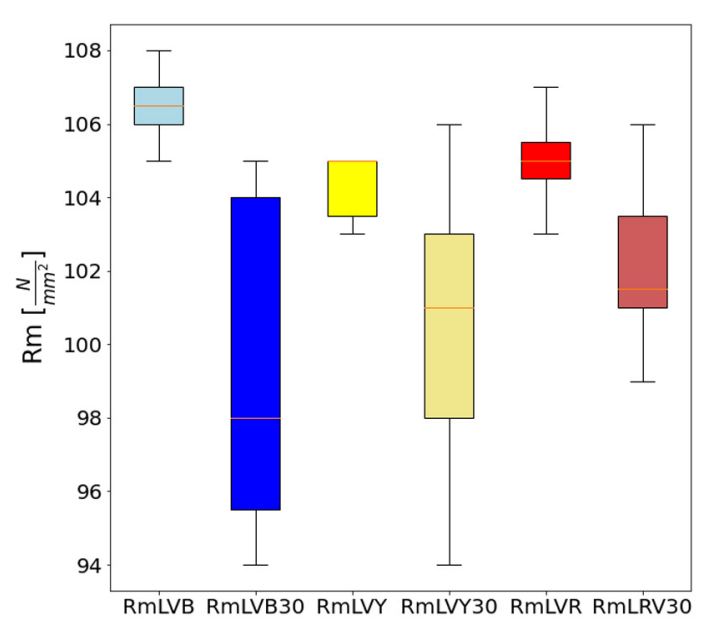

Fig. 30. Comparison of the various materials during the artificial ageing process in the nondegraded state and following 30 days of the degradation process

The box plot in Fig. 29 reveals a decrease in the ultimate strength stress between the nondegraded and degraded materials in the LV orientation of around $5 \%$. The differences between the materials are shown in Fig. 30. VeroBlue evinced the highest ultimate strength stress value and the most significant decrease during degradation, i.e., around $8 \%$ whereas, the degradation process led to decreases in the ultimate strength stress for the VeroMagenta and VeroYellow of less than $4 \%$.

\section{Conclusion}

The study revealed that the strength of PolyJet additively manufactured products depends on the direction of printing. The main weaknesses of all the PolyJet printed products related to the vertically printed components $(\mathrm{ZYX})$, the reason for which lies in the principles of this technology, i.e., the weakest point of construction is between the layers of the cured acrylicbased photo-polymeric material.

The Stratasys data sheet [10] for Vero materials indicates no differences concerning the printing method, and an ultimate strength value of between $50-60 \mathrm{MPa}$ is prescribed for all the printing directions. However, we determined an ultimate tensile strength for the vertically printed samples of nearly $50 \%$ lower than that suggested by the Stratasys data sheet. Nevertheless, in terms of low deformation and linear elasticity, it can be stated that the materials behaved isotropically regardless of the printing orientation, as indicated by the unremarkable differences in the modulus of elasticity.

With respect to printed products, it is recommended that the construction be loaded with the printing direction aligned to the orientation of the loading forces. The optimum printing method 
was determined as the L direction (YXZ). A number of conclusions were drawn from the results of the artificial ageing of thermoplastic polymers. The first samples measured after 15 days of UV exposure evinced favourable mechanical property results in certain cases, especially in terms of the stability and dispersion of the measured results. This may have been caused by the insufficient drying of the samples. The question remains whether, and to what extent, the humidity of the support material affects the properties of the polymer. This could not be evaluated since the masses of the specimens were not recorded before and after the artificial ageing process. Better results may have been attained if the experimental programme had included the measurement of the humidity in the polymer following printing and after the drying out of the material.

A further conclusion concerned the fact that the comparison of the distributions indicated the lowest difference for the horizontal L orientation (YXZ). In contrast, the vertical S orientation (ZYX) evinced the worst results according to the post-exposure comparison, i.e., a decrease in the ultimate strength stress of almost $33 \%$. The main result from the measurement of the artificial ageing of the 3D printed materials related to the discovery that the samples printed in the horizontal L orientation (YXZ), even following exposure to the UV lamp, could be used generally across all areas provided that loading was not in the $\mathrm{S}$ orientation (ZYX). A knowledge of the properties of the structure and the material is crucial in this situation.

However, with respect to the compression specimens, it could not be determined whether the differences were slight or significant. The use of an external strain gauge would, in hindsight, have been significantly more effective in terms of evaluating the differences in the modulus of elasticity. The main difference concerned the distribution for all the orientations with respect to the comparison of the ultimate tensile strength of the two differing printing approaches.

\section{Acknowledgements}

Financial support was provided for this study by the Grant Agency of the Czech Technical University in Prague; grant no. SGS21/151/OHK2/3T/12.

\section{References}

[1] Barclift, M.W., Williams, C. B., Examining variability in the mechanical properties of parts manufactured via PolyJet direct 3D printing, Proceedings of the 23rd Annual International Solid Freeform Fabrication Symposium (SFF 2012) - An Additive Manufacturing Conference, Austin, Texas, 2012, pp. 876-819.

[2] Bennett, J., Measuring UV curing parameters of commercial photopolymers used in additive manufacturing, Additive Manufacturing 18 (2017) 203-212.

https://doi.org/10.1016/j.addma.2017.10.009

[3] Blanco, D., Pelayo, F., Noriega, A., Nonisotropic experimental characterization of the relaxation modulus for PolyJet manufactured parts, Journal of Materials Research 29 (17) (2014) 1876-1882. https://doi.org/10.1557/jmr.2014.200

[4] ČSN EN ISO 4892-3:2006, Plastics - Methods of exposure to laboratory light sources - Part 3: Fluorescent UV lamps, Standard, Czech Standards Institute, 2006. (in Czech)

[5] ČSN EN ISO 527-1:2012. Plastics - Determination of tensile properties - Part 1: General principles, Standard, Czech Standards Institute, 2012. (in Czech)

[6] Gay, P., Blanco, D., Pelayo, F., Noriega, A., Fernández, P., Analysis of factors influencing the mechanical properties of flat PolyJet manufactured parts, Procedia Engineering 132 (2015) 70-77. https://doi.org/10.1016/j.proeng.2015.12.481 
[7] Mohan, N., Senthil, P., Vinodh, S., Jayanth, N., A review on composite materials and process parameters optimisation for the fused deposition modelling process, Virtual and Physical Prototyping 12 (1) (2017) 47-59. https://doi.org/10.1080/17452759.2016.1274490

[8] Prakash, K. S., Nancharaih, T., Rao, V. S., Additive manufacturing techniques in manufacturing An overview, Materials Today: Proceedings 5 (2) (2018) 3873-3882. https://doi.org/10.1016/j.matpr.2017.11.642

[9] Pugalendhi, A., Ranganathan, R., Ganesan, S., Impact of process parameters on mechanical behaviour in multi-material jetting, Materials Today: Proceedings (2020) 1-6. https://doi.org/10.1016/j.matpr.2019.12.106

[10] Stratasys: Polyjet materials, https://www.stratasys.com/polyjet-technology.

[11] Yousif, E., Haddad, R., Photodegradation and photostabilization of polymers, especially polystyrene: Review, SpringerPlus 2 (2013) No. 398. https://doi.org/10.1186/2193-1801-2-398 